Gut, 1965, 6, 448

\title{
A study of the prevalence and clinical pattern of non-specific ulcerative colitis in northern India
}

\author{
B. N. TANDON, A. K. MAThUR, L. N. MOHAPATRA, H. D. TANDON, \\ AND K. L. WIG \\ From the Gastroenterology Section of the Department of Medicine, \\ the Department of Microbiology, and the Department of Pathology, \\ All India Institute of Medical Sciences, New Delhi 16, India
}

EDITORIAL SYNOPSIS This paper indicates that non-specific ulcerative colitis is not as rare in India as has been thought previously.

A voluminous literature covering the various aspects of non-specific ulcerative colitis has been published during the last two decades and has been comprehensively covered in a symposium held under the aegis of the National Institute of Health in 1961 and in review articles by Almy (1961), Almy and Lewis (1963), Truelove and Edwards (1963), and Edwards and Truelove (1964). However, the incidence and clinical patterns of this disease in tropical countries have not been mentioned. Studies of the geographical distribution of diseases are important in reference to their pathogenesis, and reports in the literature indicate that non-specific ulcerative colitis is predominently a disease of western countries (Bockus, 1944; Bacon, 1958; Melrose, 1955). Mental stress and the strain of modern civilization might be an important causative factor (Daniels, 1948). The paucity of reports on ulcerative colitis from India suggests that it is a rare disease in that country, although several recent reports (Chaudhuri, Lahiri, Neogy, Basu, Chatterjee, Sinha, and Rai Chaudhuri 1954; Gadekar, 1962; Tandon, Upadhyaya, Tandon, and Gadekar 1964) have indicated its occurrence in north India and drawn sufficient attention to the problem for clinicians to investigate patients of the 'chronic dysentery group' as being possible cases of non-specific ulcerative colitis. This study was carried out during the last two and a half years to estimate the prevalence of non-specific ulcerative colitis in patients presenting with the complex syndrome of chronic dysentery, to study the clinical pattern of the disease in India, and to compare it with reports from western countries, in order to find out the effect of socio-economic and hygienic conditions in tropical countries on the presentation and clinical course of the disease.

\section{METHODS AND MATERIAL}

Seventy-nine patients (51 males and 28 females) who had the presenting symptoms of passing mucus and blood with loose stools were studied. Except for two patients they all belonged to the middle and poorer classes of Indian society and were exposed to the hazards of unhygienic conditions of living.

In addition to the clinical evaluation, the following investigations were carried out:-

Stools were examined to detect the presence of Entamoeba histoloytica, using two fresh routine samples after saline and iodine preparation, a warm specimen after a saline purge, a sigmoidoscopic swab specimen, and culture for amoebae in doubtful cases. To detect pathogenic bacteria, at least two stool culture studies were done by standard methods (Taylor, 1960), and serotyping of $E$. coli in 10 cases during acute exacerbations (Prakash, 1962). Sigmoidoscopic examination, barium enema studies, rectal biopsy studies, and haemoglobin estimations were also done.

\section{RESULTS}

The aetiological distribution of the 79 patients with chronic dysentery included in this study is presented

\section{TABLE I}

AETIOLOGICAL DISTRIBUTION OF 79 CASES OF CHRONIC DYSENTERY

\begin{tabular}{lrrr} 
Diagnosis & Males & Females & Total \\
\hline Non-specific ulcerative colitis & 42 & 27 & 69 \\
Chronic amoebic dysentery & 7 & 0 & 7 \\
Chronic bacillary dysentery & 2 & 1 & 3 \\
Total & 51 & 28 & 79
\end{tabular}


in Table I. Sixty-nine patients were diagnosed as suffering from non-specific ulcerative colitis and the diagnosis of specific dysenteric colitis was established in 10 patients, including three cases of bacillary dysentery and seven of chronic amoebic dysentery.

The criteria used for the diagnosis of ulcerative colitis were a clinical history of chronic dysentery uncontrolled by adequate antibacterial and antiamoebic treatment; negative results of stool studies for pathogenic bacteria and parasites; and sigmoidscopic findings of congestion, oedema, ulceration, and granularity and fragility of the mucosa of various grades. In four cases sigmoidoscopy was normal but clinical features and other studies suggested the diagnosis of non-specific ulcerative colitis.

The age and sex distribution of patients with ulcerative colitis is presented in Table II. At the time of their first visit to the clinic, the age of the youngest patient was 12 years and that of the oldest 66 years; $72.5 \%$ of the patients were between 15 and 44 years. In all age groups males were more frequently affected than females and the overall male-female ratio was $1 \cdot 5: 1$, in favour of the former.

\section{TABLE II}

DISTRIBUTION OF 69 CASES OF NON-SPECIFIC ULCERATIVE COLITIS BY AGE AND SEX

\begin{tabular}{lccc} 
Age in Years & Males & Females & Total \\
\hline $0-14$ & 2 & 0 & 2 \\
$15-24$ & 9 & 7 & 16 \\
$25-44$ & 19 & 15 & 34 \\
$45-65$ & 11 & 5 & 16 \\
$65+$ & 1 & 0 & 1 \\
Total & 42 & 27 & 69
\end{tabular}

Table III indicates the duration of dysenteric symptoms at the time of registration of the patients for the present study; $58 \%$ presented with symptoms of less than five years. The minimum period was two weeks and the maximum was 30 years.

\section{TABLE III}

DISTRIBUTION OF DURATION OF THE PRESENTING SYMPTOM OF MUCUS AND BLOOD IN STOOLS IN CASES OF ULCERATIVE COLITIS

Duration in Years No. of Cases

$\begin{array}{cc}0-4 & 40(58 \%) \\ 5-9 & 12(17 \cdot 4 \%) \\ 10-14 & 6(8.7 \%) \\ 15-19 & 8(11 \cdot 6 \%) \\ 20-24 & 1(1.4 \%) \\ 25-29 & 2(2.9 \%) \\ \text { Total } & 69(100 \%)\end{array}$

The gastrointestinal and general symptoms of these patients are presented in Tables IV and V. Blood and mucus mixed with the stools was present
TABLE IV

\begin{tabular}{clc} 
DISTRIBUTION OF & $\begin{array}{c}\text { GENERAL SYMPTOMS } \\
\text { ULCERATIVE COLITIS }\end{array}$ & 69 CASES OF \\
No. & Symptoms & No. of Cases \\
\hline 1 & Blood in stools & $69(100 \%)$ \\
2 & Mucus in stool & $69(100 \%)$ \\
3 & Loose stools & $64(92 \cdot 75 \%)$ \\
4 & Increased frequency & $64(92 \cdot 75 \%)$ \\
5 & Diffuse abdominal pain & $42(60 \cdot 8 \%)$ \\
6 & Flatulence & $34(46 \cdot 4 \%)$ \\
7 & Tenesmus & $30(43 \cdot 5 \%)$ \\
8 & Gastrocolic reflex & $23(30 \cdot 3 \%)$ \\
9 & Anorexia & $22(30 \cdot 2 \%)$ \\
10 & Vomiting & $11(15 \cdot 9 \%)$ \\
11 & Pus in stools & $9(13 \cdot 4 \%)$ \\
12 & Rectal itching & $6(8 \cdot 7 \%)$
\end{tabular}

TABLE V

DISTRIBUTION OF GENERAL SYMPTOMS IN 69 CASES OF ULCERATIVE COLITIS

\begin{tabular}{ll} 
Symptom & No. of Cases \\
\hline Weight loss & $43(62 \cdot 3 \%)$ \\
Pallor & $36(52 \cdot 1 \%)$ \\
Fever & $30(43 \cdot 5 \%)$ \\
Arthralgia & $16(23 \cdot 2 \%)$
\end{tabular}

in all, and increased frequency with loose stools was reported by $93 \%$ of the patients. Abdominal pain, flatulence, and tenesmus were frequent complaints, present in almost half of the patients. Other gastrointestinal symptoms noted were anorexia, hyperactive gastrocolic reflex, vomiting, and rectal itching. Gradual weight loss, progressive pallor, and fever during the periods of exacerbation were recorded in the majority of cases. Although pain in the smaller joints was reported by 16 patients, none had noticeable swelling of the joints at any time.

The physical examination was unrewarding. Nonspecific findings suggestive of chronic malnutrition, diffuse abdominal tenderness, and palpable descending colon were recorded. None had any significant hepatomegaly.

Anaemia of varying degree, with haemoglobin levels below $10 \mathrm{~g} . \%$, was found in 31 out of the 65 patients in whom this investigation was carried out (Table VI).

TABLE VI

DISTRIBUTION OF HAEMOGLOBIN LEVELS IN 65 CASES OF ULCERATIVE COLITIS ${ }^{1}$

\begin{tabular}{ll} 
Haemoglobin $(g . \%)$ & No. of Cases \\
\hline Less than 5.9 & $3(4.6 \%)$ \\
6 to 7.9 & $17(26.2 \%)$ \\
8 to 9.9 & $11(16.9 \%)$ \\
10 to 11.9 & $20(30 \cdot 8 \%)$ \\
$12+$ & $14(21.5 \%)$ \\
Total & $65(100 \%)$
\end{tabular}

${ }^{1}$ Haemoglobin was not estimated in four cases. 
Microbiological and parasitological studies of the stools failed to reveal pathogenic organisms in any of them.

Sigmoidoscopic appearances were recorded as stage 1 , stage 2 , and stage 3 changes, according to Bacon's classification (1958). As indicated in Table VII, 39 patients had a severe degree of diffuse hyperaemia, oedema, ragged ulceration, granularity and marked fragility of the mucosa, corresponding to the stage 3 changes. Six patients in this group had pseudopolypi. Moderate degrees of mucosal abnormality, corresponding to stage 2 changes, were recorded in 12 patients, and milder stage 1 findings were observed in 14. Endoscopic examination revealed apparently normal mucosa in four patients.

Barium enema studies were done in 51 patients (Table VIII and Fig. 1), and rectal biopsy studies in all of them.

\section{TA BLE VII}

SIGMOIDOSCOPIC FINDINGS IN ULCERATIVE COLITIS ${ }^{1}$

\begin{tabular}{ll} 
Type of Change $^{2}$ & No. of Cases \\
\hline Stage 3+ & $39(56 \cdot 5 \%)$ \\
Stage 2 & $12(17 \cdot 4 \%)$ \\
Stage 1 & $14(20 \cdot 3 \%)$ \\
Normal & $4(5 \cdot 8 \%)$ \\
Total & $69(100 \%)$
\end{tabular}

${ }^{1}$ Pseudo-polyps were seen in six cases and carcinoma was diagnosed in one case of this group.

${ }^{2}$ Classified according to Bacon's description.

\section{TABLE VIII}

DISTRIBUTION OF BARIUM ENEMA FINDINGS IN 51 PATIENTS OF ULCERATIVE COLITIS BY EXTENT OF THE DISEASE ${ }^{1}$

Extent of the Disease

Sigmoid colon

Descending and sigmoid colon

Transverse, descending, and sigmoid colon

Entire colon

No abnormality

Total

${ }^{1}$ Barium enema could not be done in 18 cases. Eight cases had back wash ileitis. Pseudo-polypi were present in nine patients and fistulae were demonstrated in two cases. Carcinomatous complication was recorded in two patients.

\section{TABLE IX}

DISTRIBUTION OF COMPLICATIONS IN 69 CASES OF ULCERATIVE COLITIS ${ }^{1}$

\begin{tabular}{ll} 
Complication & No. of Cases \\
\hline Haemorrhage & $6(8.7 \%)$ \\
Carcinoma & $2(2.9 \%)$ \\
Fistulous tract & $2(2.9 \%)$ \\
Perforation $^{2}$ & $1(1.5 \%)$
\end{tabular}

${ }^{1}$ All these complications were recorded in patients grouped as the severe form of ulcerative colitis.

${ }^{2}$ Clinical diagnosis.

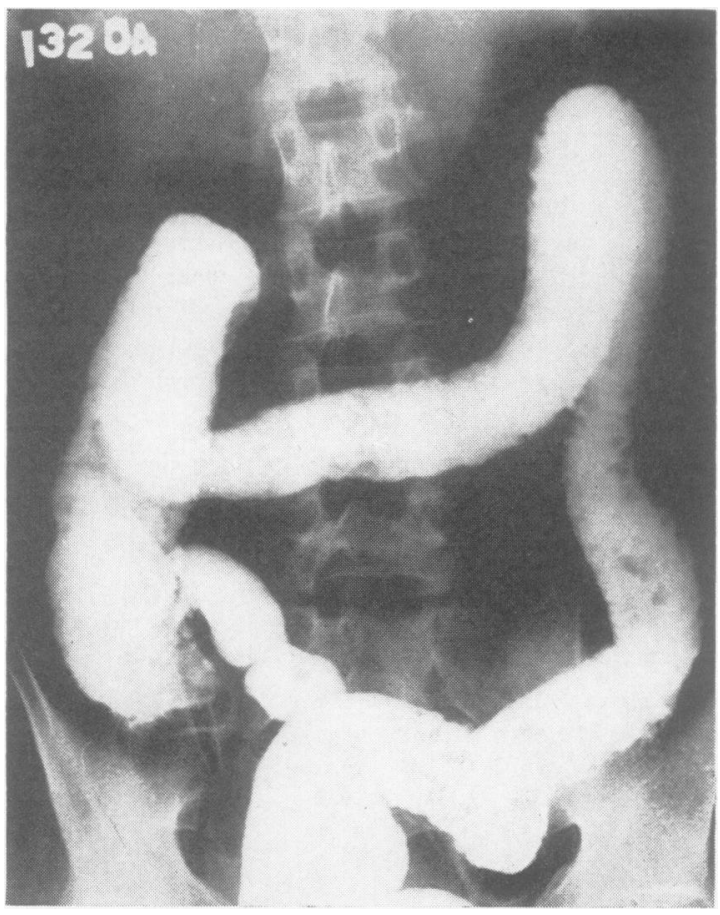

FIG. 1. Representative barium enema findings in a patient with extensive ulcerative colitis with destruction of mucosa, marginal serrations, and pseudo-polyposis.

Serious complications of ulcerative colitis were recorded in 11 patients. As indicated in Table IX, severe rectal haemorrhage was present in six, carcinoma of the colon was confirmed by radiology and histology in two patients, and fistulous tracts were demonstrated by $x$-ray studies in two. Perforation as the cause of death was clinically diagnosed in one patient.

Systemic complications of ulcerative colitis were not recorded in any. Seven patients with ulcerative colitis in the present series succumbed to the disease, carrying the mortality of $10 \%$. Two patients died while on a medical régime, which included A.C.T.H. therapy; one of them had clinical features of perforation and the cause of death was not apparent in the other. Five patients died in the post-operative period. Two had inoperable carcinoma complicating ulcerative colitis and they expired due to malignant cachexia four and six weeks after the palliative colostomy. The cause of death in the remaining three, who had subtotal colectomy because the condition did not respond to medical management, was peritonitis, septicaemia, and irreversible shock in the post-operative period.

On the basis of clinical data and the results of investigations, the patients can be classified into two 
groups, severe and mild or quiescent. Thirty-nine patients had ulcerative colitis in its severe form. Thirty-six cases in this group presented with progressively deteriorating dysenteric and general symptoms and they had stage 3 mucosal changes on sigmoidoscopic examination. Two patients presented with severe anaemia (haemoglobin levels of $4 \mathrm{~g} . \%$ and 5.5 g. $\%$ ) and stage 2 sigmoidoscopic findings. Their dysenteric symptoms were in remission at the time of study. A barium enema showed that in one of them the entire colon was involved. The remaining patient of this group was in complete clinical remission. He had stage 2 sigmoidoscopic findings but a barium enema revealed disease of the entire colon with pseudo-polypi. All the complications and mortality recorded in the present series were observed in this group of severely ill patients. During the period of investigation, four patients of this group went into natural remission, the rest requiring A.C.T.H., steroid, or Salazopyrin to control the symptoms. Surgical treatment had to be carried out in eight, either because they did not respond to medical management or on account of some complication of the disease.

Thirty patients belonged to the group of ulcerative colitis of a milder form. The clinical features of these patients were the same as of the severe type, but of a milder degree. Sigmoidoscopic findings revealed stage 3 changes in three patients, stage 2 in nine, and stage 1 in 14. Three of the four patients with normal findings on sigmoidoscopy had evidence of segmental colitis of the sigmoid colon on barium enema studies. None of the patients of this group had any of the complications of ulcerative colitis and none had a fatal end. Symptoms were easily controlled by nonspecific measures. Salazopyrin was used only in one case and the response was gratifying, but because Salazopyrin was not available, sulphasuxidine was added to the non-specific therapy in the remainder. None required steroid therapy or surgical intervention. Three patients of this group in a follow-up period of three to six months presented with features of acute exacerbations. Steroids controlled the symptoms in two of them and surgery was undertaken in the remaining case. This patient had a fatal postoperative course, and a resected colon specimen showed classical gross and histological features of ulcerative colitis.

\section{DISCUSSION}

Classical clinical features, characteristic sigmoidoscopic findings, and the absence of pathogens in the stools of the patients diagnosed as ulcerative colitis in the present series conform with the features of the

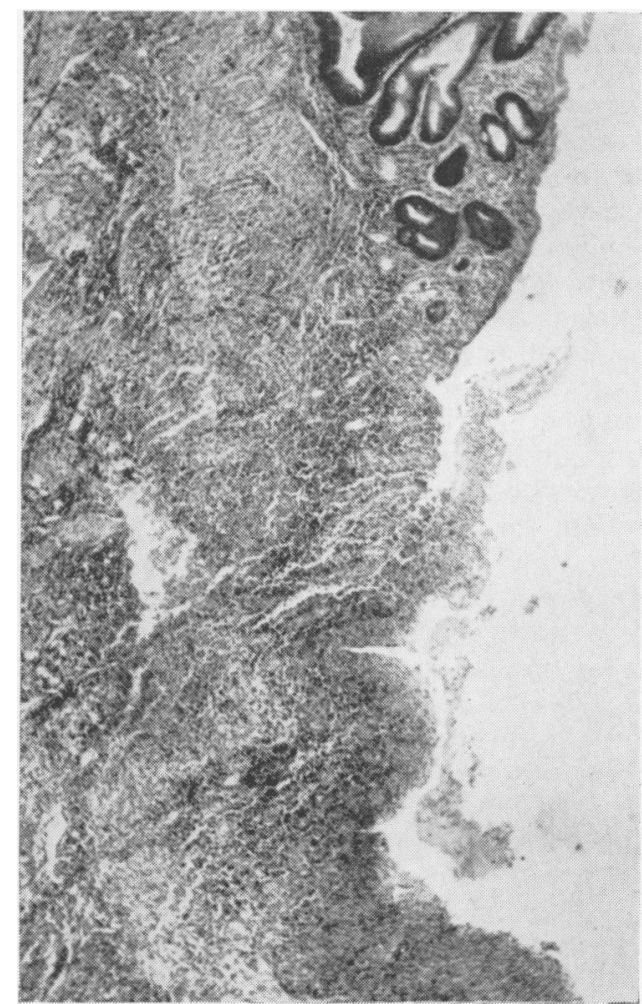

FIG. 2. Characteristic histological appearance of nonspecific ulcerative colitis (haematoxylin and eosin $\times 45$ ).

proven cases of this disease as reported in the literature (Truelove and Edwards, 1963; Bacon, 1958). Resected colon specimens of operated cases of ulcerative colitis of the present series had gross and microscopic (Fig. 2) changes diagnostic of this disease, thus further establishing the validity of the criteria used for the diagnosis of ulcerative colitis in this study. The possibility of chronic bacillary dysentery and chronic amoebic colitis was excluded in these patients by the absence of pathogens in the stools even during acute exacerbations. A lack of therapeutic response, or even worsening of the disease, on adequate antibacterial and antiamoebic therapy also indirectly favoured the diagnosis of ulcerative colitis.

Of the patients with chronic dysentery, $87 \cdot 3 \%$ had non-specific ulcerative colitis. This rather high incidence of the disease does not indicate its true prevalence in the population at large in this part of India. Patients suffering from a specific dysentery, amoebic and bacillary, are successfully treated by the general practitioner and the internist, but when such symptoms are due to non-specific ulcerative colitis patients frequently have to be referred to the speciality 
clinic. The interest in non-specific ulcerative colitis at this centre attracted cases of this disease from nearby regions for investigation and management, and these factors have provided highly selected material for the study and have magnified the incidence of ulcerative colitis in this report. However, the importance of ulcerative colitis as a cause of the chronic dysentery syndrome in this part of the country is sufficiently established to warrant the same attention as in western countries, although its true incidence in different parts of India needs to be worked out. Its recognition and early diagnosis is of paramount importance, even in a tropical country where amoebic and bacillary infections of the bowel are more frequent causes of dysentery, as in the modern era of effective anti-amoebic and antibacterial therapy morbidity and mortality associated with specific forms of dysentery are significantly less than are found in non-specific ulcerative colitis (Craig, 1947; Lancet, 1964).

The clinical features of ulcerative colitis noted in the present study are similar to those reported in the western literature. Of the patients studied, $56.5 \%$ had the fulminant type and $43.5 \%$ had the insidious or mild type of this disease. Such a high proportion of the mild type of disease has not been reported in any series from the western countries. This observation on the clinical pattern of ulcerative colitis in India needs due consideration in any study of its pathogenesis and acute exacerbations in that country. It also indicates that low socio-economic status and poor hygienic conditions had no adverse effects on the clinical progress of the patients.

Of the patients presenting, $56.5 \%$ had sigmoidoscopic appearances characteristically described in the severe forms of the disease, that is, the third stage of Bacon's classification, and $17 \%$ and $20 \%$ of the cases had first and second stage changes respectively. There was good correlation between the severity of the disease according to sigmoidoscopic findings and its clinical presentation. Limitations of sigmoidoscopy in the diagnosis of segmental colitis and ulcerative colitis in complete remission were noted in three patients and one patient respectively. The diagnostic value of sigmoidoscopic findings in tropical countries is also limited because of the similarity between stage 1 and stage 2 changes with the appearances described in chronic amoebic and chronic bacillary dysentery and stage 3 changes in acute bacillary dysentery (Manson-Bahr, 1943). In addition to sigmoidoscopic changes, proper studies of the stools, including culture examination and the results of therapeutic trials, must be given due consideration in the diagnosis of non-specific ulcerative colitis.

The utility of barium enema studies to define the extent of the disease, to detect the complications, and for the diagnosis of segmental ulcerative colitis as reported in the literature was confirmed in the present study. The limitation of this investigation when the disease is localized in the rectum and rectosigmoid region was observed in $20 \%$ of the cases.

Complications of ulcerative colitis were recorded in $16 \%$ of the patients, and comparable figures from western countries are $31 \%$ to $40 \cdot 1 \%$ (Ricketts and Palmer, 1946; Rankin, Bargen, and Buie, 1932). Pseudo-polyposis was observed in $17.6 \%$ of the radiologically studied cases. The incidence of pseudopolypi has ranged from $10 \%$ to $32.5 \%$ in the reports of Rankin et al. (1932) and Bacon (1958) respectively. Fistulae were recorded in $2.9 \%$ of the cases compared with reported series of 6 to $43.5 \%$ (Bacon, 1958; Sloan, Bargen, and Gage, 1950). Perforation is the most serious complication of this disease, and was thought to be the cause of death in one patient of the present series. Reports of the incidence of this complication in the literature vary from 2.6 to $43 \%$ (Sloan et al., 1950; Bargen, 1943). Carcinoma was recorded in two cases $(2.9 \%)$ in the present series compared with the incidence of $3.5 \%$ reported by Truelove and Edwards (1963).

Study of ulcerative colitis in tropical countries offers a special opportunity to investigate the role of bacillary and amoebic infection in the pathogenesis of this disease and as a cause of acute exacerbations. Since the pioneer report of Hurst in 1921 many controversial papers have been published regarding the role of bacterial, parasitic, fungal, and viral infections in the pathogenesis of non-specific ulcerative colitis (Felsen and Gorenberg, 1936; Palmer, 1948; Bacon, 1958; Syverton, 1961). In the present study none of the patients diagnosed as ulcerative colitis showed the presence of Entamoeba histolytica or pathogenic bacteria on repeated examination of stools. Such observations from a tropical country, with its wide prevalence of bacillary and amoebic dysentery, negates any direct role of these pathogens in the manifestations of non-specific ulcerative colitis.

\section{SUMMARY AND CONCLUSIONS}

Chronic non-specific ulcerative colitis was diagnosed in 69 cases, in the two and a half year period of this study. Clinical features, microbiological studies of the stools, and sigmoidoscopic findings in these patients conformed to descriptions for this disease from the western countries. The severe form of the disease was recorded in $56.5 \%$, while $43.5 \%$ of the patients were in the mild or quiescent stage of ulcerative colitis. Complications of a serious nature, but localized to the colon, were observed in $16 \%$ of 
the patients. None had significant systemic complications. Ten per cent of the patients died. In none of the cases, could any positive contributory role of pathogenic bacteria of the colon or Entamoeba histolytica be demonstrated in the pathogenesis or manifestations of ulcerative colitis. Similarly the socio-economic and hygienic conditions of the country did not seem to have any adverse effect on the course of ulcerative colitis.

This report establishes the importance of chronic non-specific ulcerative colitis even in a tropical country where specific forms of colitis are more frequent problems. The current evidence casts serious doubts on the prevailing belief that this disease is very rare in India.

This study was supported in part by the Indian Council of Medical Research.

\section{REFERENCES}

Almy, T. P. (1961). Ulcerative colitis. Gastroenterology, 41, 391-400. nd Lewis, C. M. (1963). Ulcerative colitis: a report of progress, based on recent literature. Ibid., 45, 515-528.

Bacon, H. E. (1958). Ulcerative Colitis. Lippincott, Philadelphia.

Bargen, J. A. (1943). The Modern Management of Colitis, Thomas, Springfield, Illinois.

Bockus, H. L. (1944). Gastroenterology, vol. 2, p. 573. Saunders, Philadelphia.

Chaudhuri, R. N., Lahiri, D. C., Neogy, K. N., Basu, S. P., Chatterjee, S. N., Sinha, G., and Rai Chaudhuri, M. N. (1954). Studies on ulcerative colitis. Bull. Calcutta Sch. trop. Med., 1, 9-11.
Craig, C. F. (1947). Amebiasis. In Oxford Medicine, edited by H. A. Christian, vol. V, Sect. 816, p. 46. Oxford University Press, New York.

Daniels, G. E. (1948). Psychiatric factors in ulcerative colitis. Gastroenterology, 10, 59-62.

Edwards, F. C., and Truelove, S. C. (1964). The course and prognosis of ulcerative colitis (Parts III \& IV). Gut, 5, 1-22.

Felsen, J., and Gorenberg, H. (1936). Chronic dysentery, distal ileitis and ulcerative colitis: follow-up of Jersey City epidemic of bacillary dysentery. Amer. J. med. Sci., 192, 553-556.

Gadekar, N. G., (1962). Ulcerative colitis: incidence and diagnosis. Indian J. Radiol., 16, 151-166.

Hurst, A. F. (1921). Ulcerative colitis. Guy's Hosp. Rep., 71, 26-41.

Lancet (1964). (Editorial). Prognosis in ulcerative colitis. 1, 368-369.

Manson-Bahr, P. (1943). The Dysenteric Disorders, 2nd ed., p. 433. Cassell, London.

Melrose, A. G. (1955). The geographical incidence of ulcerative colitis in Great Britain. Gastroenterology, 29, 1055-1060.

Palmer, W. L. (1948). Chronic ulcerative colitis. Ibid., 10, 767-781

Prakash, O. (1962). Escherichia coli serotypes isolated from sporadic cases of diarrhoea and gastroentritis in infants in Delhi. Indian J. med. Res., 50, 4, 607-611.

Rankin, F. W., Bargen, J. A., and Buie, L. A. (1932). The Colon, Rectum and Anus, p. 237. Saunders, Philadelphia.

Ricketts, W. E., and Palmer, W. L. (1946). Complications of chronic non-specific ulcerative colitis. Gastroenterology, 7, 55-66.

Sloan, W. P., Bargen, J. A., and Gage, R. P. (1950). Life histories of patients with ulcerative colitis: a review of 2,000 cases. Ibid., 29, 25-38.

Symposium on ulcerative colitis (1961). Ibid., 40, 286-369.

Syverton, J. T. (1961). Enteroviruses. Ibid., 40, 331-337.

Tandon, B. N., Upadhyaya, A. K., Tandon, H. D., and Gadekar, N. G. (1964). Ulcerative colitis in northern Indian subjects. (A preliminary report.) J. Ass Phycns, India, 12, 43-52.

Taylor, J. (1960). Laboratory Diagnosis of enteric infections. Enteropathogenic Escherichia coli. In Recent Advances in Clinical Pathology, Series III, edited by S. C. Dyke. Churchill, London.

Truelove, S. C., and Edwards, F. C. (1963). The course and prognosis of ulcerative colitis (Parts I \& II). Gut, 4, 299-315. 